удК 519.626

Е. В. Аксенюшкина

Байкальский государственный университет, 2. Иркутск, Российская Федеращия

\title{
НАХОЖДЕНИЕ ОПТИМАЛЬНОЙ ИНВЕСТИЦИОННОЙ СТРАТЕГИИ ФИНАНСОВОЙ ОРГАНИЗАЦИИ
}

\begin{abstract}
АНнотАцИя. В статье проводится полный анализ экономической ситуации, связанной с построением инвестиционной политики финансовой организации. В представленном исследовании рассмотрены все ситуации, связанные с ограниченными возможностями финансовой организации по наращиванию собственных средств, а также с учетом периода планирования деятельности. Такой анализ позволяет построить более полную картину инвестиционной политики фирмы. Для нахождения оптимального поведения с целью получения наибольшего объема финансовых средств, построена задача оптимального управления. На основе решения серии задач линейного программирования выстраивается фундамент для использования принципа максимума Понтрягина. Такой подход дает возможность построить сильно экстремальные управления в исследуемой задаче, что дает возможность получить оптимальную инвестиционную стратегию организации.

кЛючЕВЫЕ словА. Сильно экстремальное управление; оптимальное управление; инвестиционная политика; функция Понтрягина; линейное программирование. ИНФОРМАЦИЯ О СТАТЬЕ. Дата поступления 30 октября 2017 г.; дата принятия к печати 19 декабря 2017 г.; дата онлайн-размещения 29 декабря 2017 г.
\end{abstract}

Ye. V. Aksenyushkina Baikal State University, Irkutsk, Russian Federation

\section{FINDING AN OPTIMAL INVESTMENT STRATEGY OF FINANCIAL INSTITUTION}

\begin{abstract}
The article provides the full analysis of the economic situation associated with constructing the investment policy of a financial institution. The study examines all situations related to limited capacity of the financial institution for increasing its own funds, as well as takes into account the period of activity planning. This analysis allows building the more complete picture of a company's investment policy. The optimal control problem is formed in order to find an optimal behavior for obtaining the greatest amount of financial resources. The foundation for using the Pontryagin maximum principle is constructed on a basis of the series of linear programming problems solution. This approach makes it possible to construct the highly extreme control in the research problem, which allows to obtain the optimal investment strategy of the institution.
\end{abstract}

KEYWORDS. Strongly extreme management; optimal management; investment policy; Pontryagin's function; linear programming.

ARTICLE INFO. Received October 30, 2017; accepted December 19, 2017; available online December 29, 2017.

Рассмотрим модель инвестиционного поведения финансовой организации в виде задачи оптимального управления.

Пусть $[0 ; T]-$ период планирования; $x(t)$ - собственные средства финансовой организации в момент $t \in[0, T] ; v(t)$ - доля собственных средств, идущая на инвестиции в момент $t$; $u(t)$ - отношение привлеченных средств к собственным в момент $t$. Подобные модели уже были рассмотрены $[1 ; 2]$

(C) E. В. Аксенюшкина, 2017

\section{Baikal Research Journal}

электронный научный журнал Байкальского государственного университета 


$$
\begin{gathered}
\Phi(u, v)=\int_{0}^{T} e^{-\rho t}(1-u(t)-v(t)) x(t) d t \rightarrow \max , \\
\dot{x}=r(c u+v) x, \quad x(0)=x_{0}, \\
W=\{(u(\cdot), v(\cdot)) \in P C[0 ; T]:(u(t), v(t)) \in V, \quad t \in[0 ; T]\},
\end{gathered}
$$

где $V=\left\{u(t) \geq 0,0 \leq v(t) \leq 1, c u(t)+v(t) \leq \frac{g}{r}, t \in[0, T], c \in[0,1), \quad r<\rho\right\}$.

В рамках рассматриваемой модели $r \in(0,1]$ - процентная ставка по инвестициям; $c=1-r_{2}, 0 \leq c \leq 1$, где $r_{2} \in(0,1]-$ процентная ставка по кредиту. Величина $g=$ const $>0$ характеризует верхнюю границу темпа прироста собственных средств финансовой организации, что подчеркивает ее ограниченные возможности по наращиванию собственных средств.

Проведем исследование полученной задачи на основе принципа максимума Понтрягина $[3 ; 4 ; 5 ; 6]$.

Функция Понтрягина для построенной задачи будет иметь вид

$$
H(\psi, x, u, v)=\psi r(c u+v) x+(1-u-v) x .
$$

С учетом этого сопряженное уравнение запишем в виде

$$
\dot{\psi}=\rho \psi-H_{x}, \quad \psi(T)=0 .
$$

Построим пару сильно х-экстремальных управлений

$$
(\bar{u}(t), \bar{v}(t))=\arg \max _{u, v \in V} H(\psi(t, \bar{u}, \bar{v}), x(t), u, v), \quad t \in[0 ; T],
$$

где $x(t) \in X-$ произвольная фазовая траектория из множества всех фазовых траекторий задачи. Подставим значение функции Понтрягина:

$$
(\bar{u}(t), \bar{v}(t))=\arg \max _{u, v \in V}(r \psi(t, \bar{u}, \bar{v})(c u+v)-u-v) x(t),
$$

так как $x(t) \geq 0$. С учетом этого можно утверждать, что сильно х-экстремальные управления будут оптимальными в рассматриваемой задаче $[7,8]$.

Таким образом, для поиска оптимальных управлений необходимо решить следующую задачу

$$
((\psi r c-1) u+(\psi r-1) v) x \rightarrow \max , \quad u \in V, v \in V
$$

Поскольку $x(t) \geq 0$, то задача на поиск управлений $u^{*}(t)$ и $v^{*}(t)$ эквивалентна задаче

$$
\begin{gathered}
y_{1} u+y_{2} v \rightarrow \max , \\
c u+v \leq \frac{g}{r}, \\
u \geq 0, \quad v \in[0,1],
\end{gathered}
$$

где

$$
\begin{aligned}
& y_{1}(\psi)=r \psi c-1, \\
& y_{2}(\psi)=r \psi-1 .
\end{aligned}
$$

\section{Baikal Research Journal}


Полученная задача является задачей линейного программирования с двумя переменными, поэтому ее можно решить графически $[9 ; 10 ; 11]$. Построив допустимое множество, видим, что задача распадается на два случая в зависимости от конкретных начальных условий.

Поскольку особенностью задач линейного программирования является то, что наибольшее значение целевой функции достигается в вершине (угловой точке) допустимого множества, то в основе анализ построенной задачи лежит определение расположения целевого вектора.

Проанализируем задачу (1) в зависимости от значений $y_{1}$ и $y_{2}$, а также отношения $\underline{g}$.

Рассмотрим случай, когда $\frac{g}{r}>1$ (случай А). Опираясь на разработанный алгоритм решения задачи (1) [12], выделим только возможные ситуации.

Случай, при котором $y_{1}=0$ и $y_{2}>0$. Решение задачи запишем в виде

$$
\left\{\begin{array}{c}
u^{*} \in\left[\begin{array}{c}
0, \frac{g-r}{r c}
\end{array}\right] . \\
v^{*}=1
\end{array}\right.
$$

Графическая иллюстрация решения представлена на рис. 1.

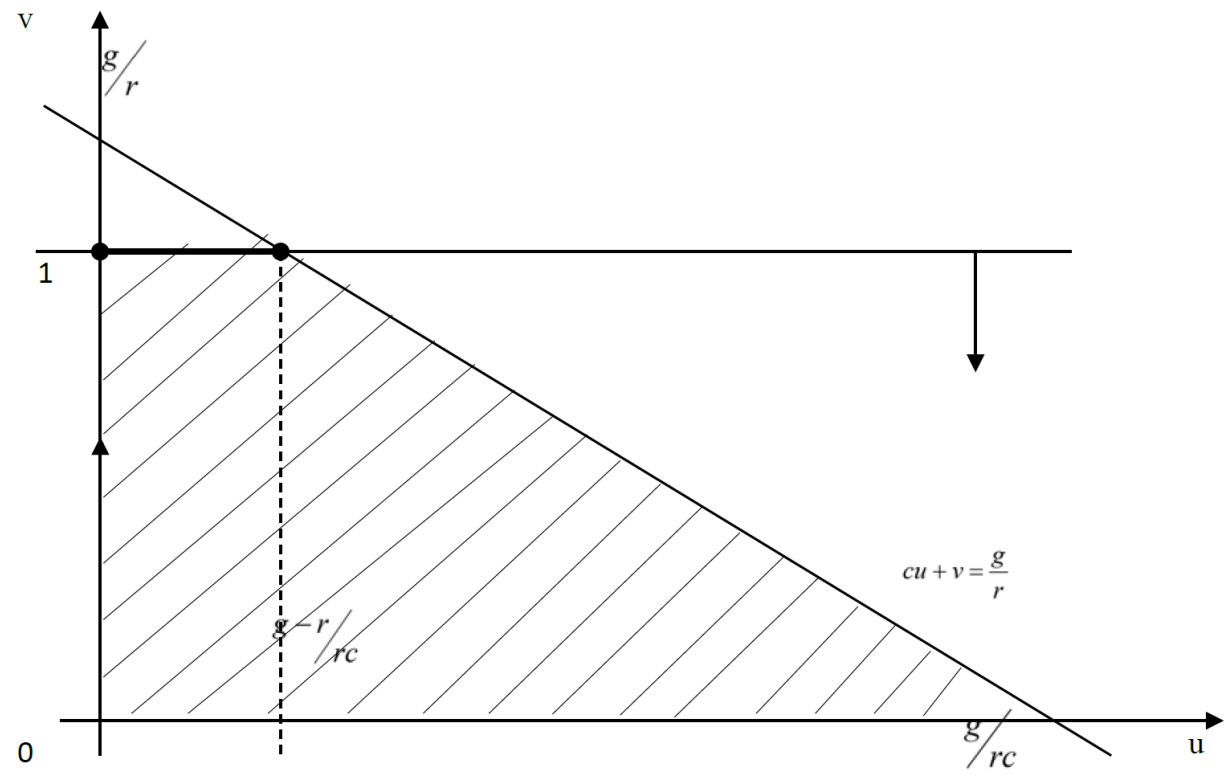

Рис. 1. Оптилальное решение задачи в случае $A$

В случае, когда $y_{1}>0$ и $y_{1}<c y_{2}$ решением задачи будет

$$
\left\{\begin{array}{c}
u^{*}=\frac{g-r}{r c} \\
v^{*}=1
\end{array} .\right.
$$

\section{Baikal Research Journal}


Графическая иллюстрация решения представлена на рис. 2.

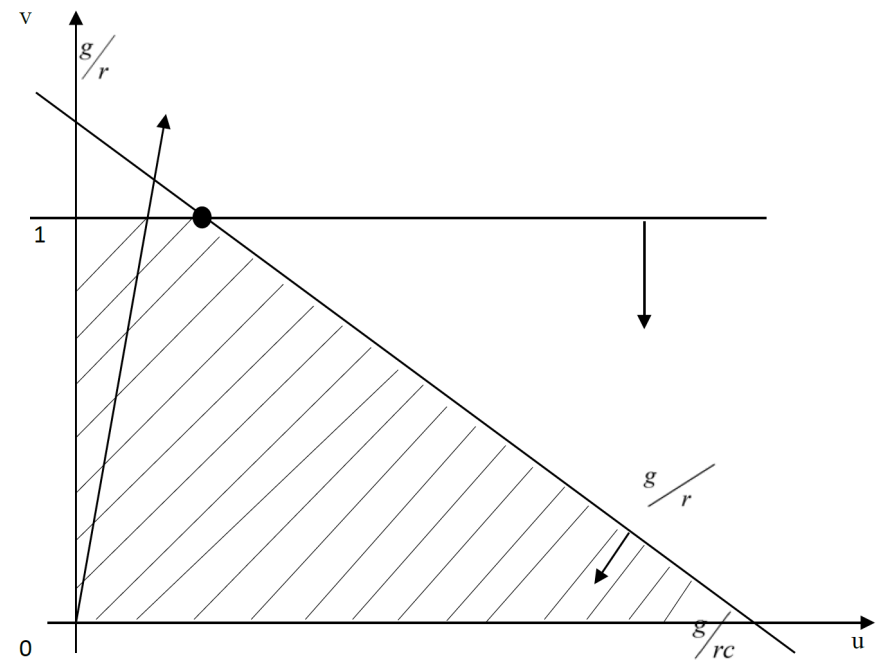

Рис. 2. Оптимальное решение задачи в случае A

В случае, когда $y_{1}<0$ и $y_{2}<0$ Очевидно, что оптимальным решением будет точка

$$
\left\{\begin{array}{l}
u^{*}=0 \\
v^{*}=0
\end{array}\right.
$$

Графическая иллюстрация решения представлена на рис. 3.

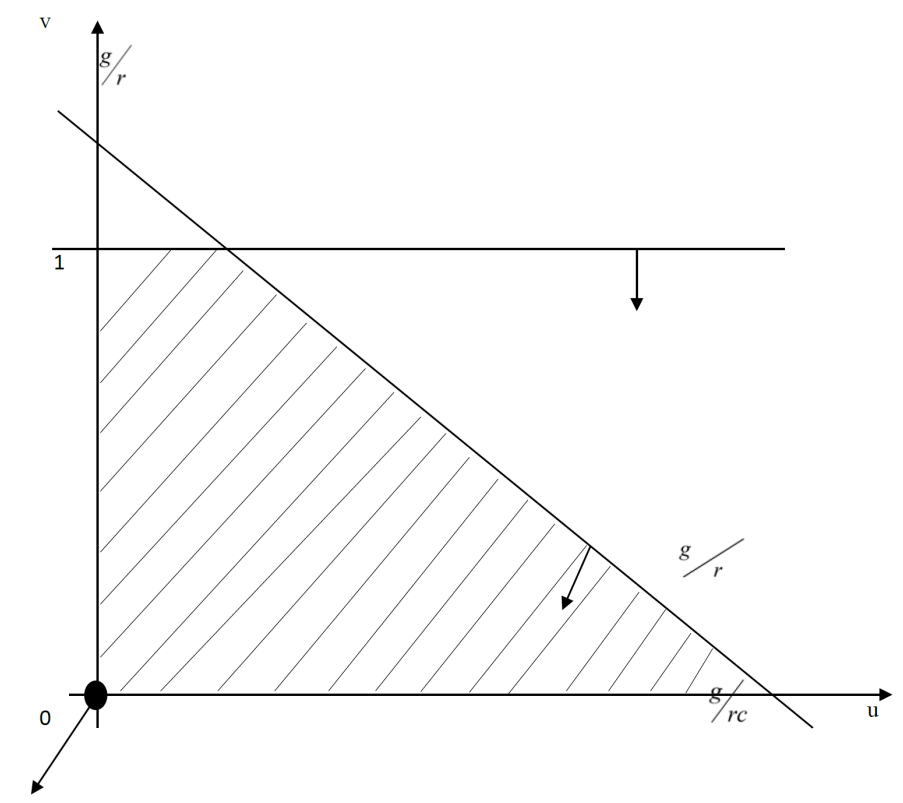

Рис. 3. Оптимальное решение задачи в случае A

\section{Baikal Research Journal}


В случае, при котором $y_{1}<0, y_{2}>0, y_{1}<c y_{2}$, решением задачи будет

$$
\left\{\begin{array}{l}
u^{*}=0 \\
v^{*}=1 .
\end{array}\right.
$$

Графическая иллюстрация решения представлена на рис. 4.

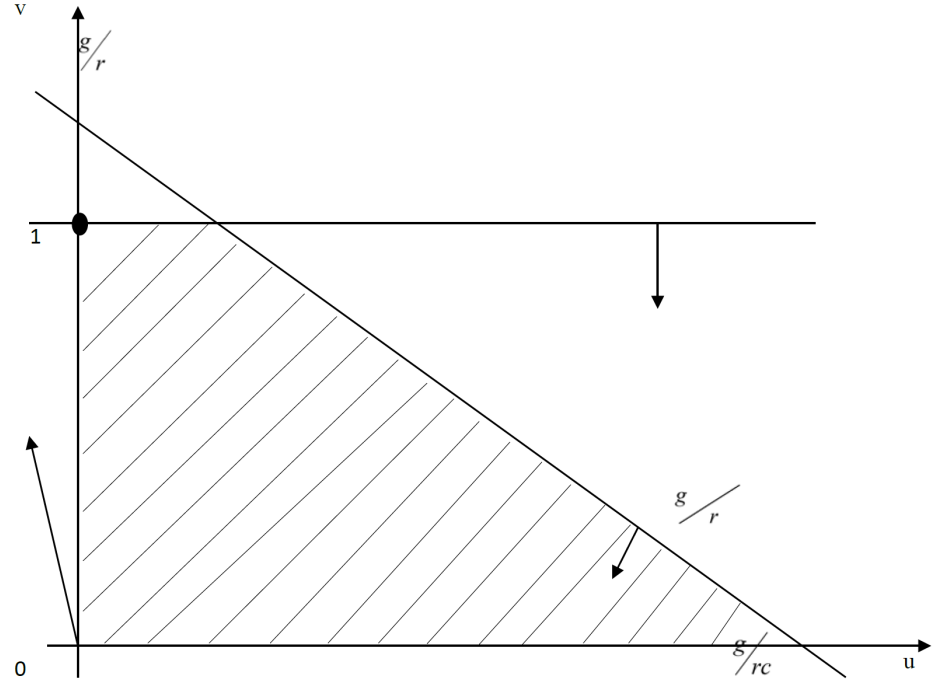

Рис.4. Оптилальное решение задачи в случае A

Пусть $y_{1}<0, y_{2}=0$, тогда решением задачи будет иметь вид

$$
\left\{\begin{array}{c}
u^{*}=0 \\
v^{*} \in[0,1] .
\end{array}\right.
$$

Графическая иллюстрация решения представлена на рис. 5.

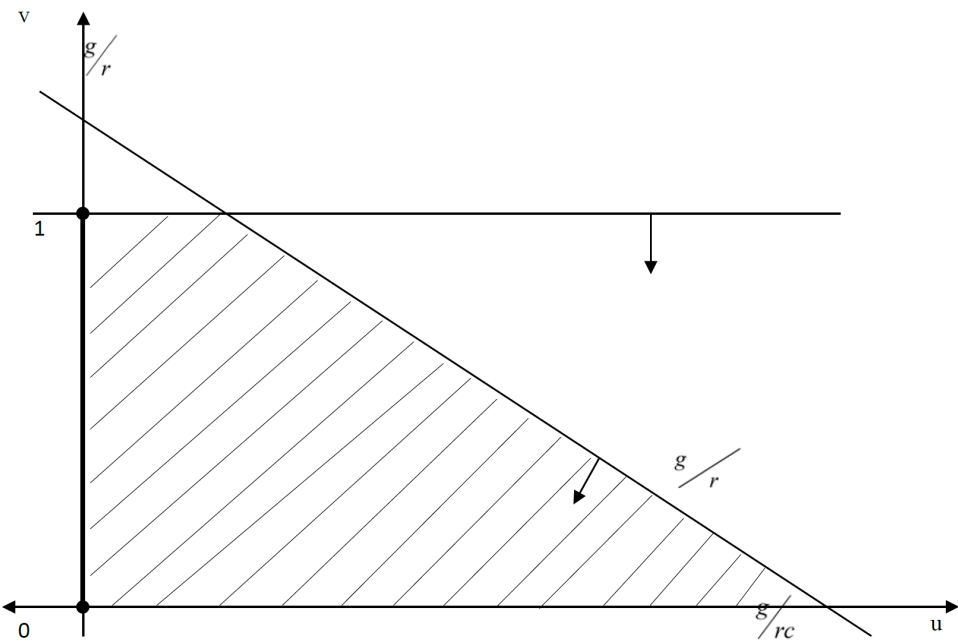

Рис. 5. Оптилальное решение задачи в случае $A$

\section{Baikal Research Journal}


Теперь анализируя все случаи получим общее решение задачи (1) (случай А)

$$
\begin{gathered}
u^{*}(\psi, t)=\left\{\begin{array}{c}
0, \quad y_{1}(\psi, t)<0 \\
{\left[\begin{array}{c}
g-r \\
r c
\end{array}\right], \quad y_{1}(\psi, t)=0,} \\
\frac{g-r}{r c}, \quad y_{1}(\psi, t)>0
\end{array}\right. \\
v^{*}(\psi, t)=\left\{\begin{array}{cc}
0, & y_{2}(\psi, t)<0 \\
{[0 ; 1],} & y_{2}(\psi, t)=0 . \\
1, & y_{2}(\psi, t)>0
\end{array}\right.
\end{gathered}
$$

В случае, когда отношение $\frac{g}{r} \leq 1$, решение задачи будет иметь вид (случай Б)

$$
\begin{gathered}
u^{*}(t)=0, \\
v^{*}(\psi, t)=\left\{\begin{array}{cc}
0, & y_{2}(\psi, t)<0 \\
{\left[0 ; \frac{g}{r}\right],} & y_{2}(\psi, t)=0 . \\
\frac{g}{r}, & y_{2}(\psi, t)>0
\end{array}\right.
\end{gathered}
$$

Представленное решение уже было получено [12].

Приступим к решению задачи А. По найденным управлениям рассчитаем сопряженную траекторию $\psi(t, u, v), t \in[0 ; T]$. Найдем значения $y_{1}$ и $y_{2}$ в конечный момент времени $T$

$$
\begin{aligned}
& \left.y_{1}(\psi(t), t)\right|_{t=T}=(r c \psi(t)-1)_{t=T}=r c \psi(T)-1=-1<0, \\
& \left.y_{2}(\psi(t), t)\right|_{t=T}=\left.(r \psi(t)-1)\right|_{t=T}=r \psi(T)-1=-1<0 .
\end{aligned}
$$

Следовательно, $u^{*}=0$ и $v^{*}=0$. Подставляем данные управления в сопряженное уравнение

$$
\dot{\psi}=\rho \psi-r \psi(c u+v)-(1-u-v), \psi(T)=0,
$$

получаем

$$
\dot{\psi}=\rho \psi-1, \quad \psi(T)=0 .
$$

Решая задачу Коши, получим общую формулу для сопряженной траектории

С учетом $\psi(T)=0$, получим

$$
\psi(t)=c_{1} e^{\rho t}+\frac{1}{\rho} .
$$

$$
\psi(t)=\frac{1}{\rho}-\frac{1}{\rho} e^{\rho(t-T)}, \quad t \leq T .
$$

Подставляем найденное решение в $y_{1}$ и $y_{2}$

\section{Baikal Research Journal}




$$
\begin{aligned}
& y_{1}(\psi(t), t)=r c \psi(t)-1=\frac{r c}{\rho}\left(1-e^{\rho(t-T)}\right)-1, \\
& y_{2}(\psi(t), t)=r \psi(t)-1=\frac{r}{\rho}\left(1-e^{\rho(t-T)}\right)-1 .
\end{aligned}
$$

Найдем точки переключения $y_{1}(\psi(t), t)=0$

$$
e^{\rho(t-T)}=\frac{r c-\rho}{r c}
$$

Прологарифмировав это выражение, получим

$$
\rho(t-T)=\ln \left(\frac{r c-\rho}{r c}\right)
$$

отсюда

$$
t=T-\frac{1}{\rho} \ln \left(\frac{r c}{r c-\rho}\right)
$$

Обозначим через $\tau_{1}=T-\frac{1}{\rho} \ln \left(\begin{array}{r}r c \\ r c-\rho\end{array}\right)$ точку переключения для $y_{1}(t)$. В точке $t=\tau_{1} \quad y_{1}(t)=0$, при $\tau_{1}<t \leq T \quad y_{1}(t)<0$.

Аналогично находим точки переключения $y_{2}(\psi(t), t)=0$

$$
e^{\rho(t-T)}=\frac{r-\rho}{r} .
$$

Прологарифмировав это выражение, получим

отсюда

$$
\rho(t-T)=\ln \left(\frac{r-\rho}{r}\right)
$$

$$
t=T-\frac{1}{\rho} \ln \left(\frac{r}{r-\rho}\right) .
$$

Обозначим через $\tau_{2}=T-\frac{1}{\rho} \ln \left(\frac{r}{r-\rho}\right)$ точку переключения. В точке $t=\tau_{2} \quad y_{2}(t)=0$, при $\tau_{2}<t \leq T \quad y_{2}(t)<0$.

Рассмотрим разность $\tau_{1}-\tau_{2}$

$$
\tau_{1}-\tau_{2}=\frac{1}{\rho}\left(\ln \frac{r}{r-\rho}-\ln \frac{r c}{r c-\rho}\right)=\frac{1}{\rho} \ln \frac{r c-\rho}{c(r-\rho)}=\frac{1}{\rho} \ln \frac{r c-\rho}{r c-\rho c} .
$$

Так как

$$
\begin{gathered}
r-\rho>0, \quad \rho>0, \\
c>0, \quad c-1<0,
\end{gathered}
$$

то получим, что соотношение

а следовательно

$$
\frac{\rho(c-1)}{r c-\rho c}<0,
$$

\section{Baikal Research Journal}




$$
\frac{r c-\rho}{r c-\rho c}<1
$$

отсюда получаем

$$
\ln \left(\frac{r c-\rho}{r c-\rho c}\right)<0
$$

Значит $\tau_{1}<\tau_{2}$.

Найдем знак $y_{2}(t)$ в зависимости от расположения $\tau_{2}$.

1) Если $\tau_{2} \leq 0$, то $y_{2}(t)<0, \quad 0 \leq t \leq T$. Значит

$$
\left\{\begin{array}{l}
u^{*}=0 \\
v^{*}=0
\end{array} \quad t \in[0 ; T] .\right.
$$

2) Если $0<\tau_{2}<T$, то сделаем предположения на знак $y_{2}(\psi)$ на участке $\left(0 ; \tau_{2}\right]$

1. $y_{2}(\psi)<0$ на $0<t<\tau_{2}$.

2. $y_{2}(\psi)=0$ на $0<t<\tau_{2}$.

3. $y_{2}(\psi)>0$ на $0<t<\tau_{2}$.

Далее проведем анализ этих ситуаций.

1. Пусть $y_{2}(\psi(t), t)<0, t<\tau_{2}$. Следовательно, $v^{*}=0$. Подставляем данное управление в сопряженное уравнение и находим его решение

$$
\begin{gathered}
\dot{\psi}=\rho \psi-1 \Rightarrow \psi(t)=c_{1} e^{\rho t}+\frac{1}{\rho}, \\
\psi\left(\tau_{2}\right)=\frac{1}{r} \Rightarrow c_{1} e^{\rho T}+\frac{1}{\rho}=\frac{1}{r} \Rightarrow c_{1}=\frac{\rho-r}{r \rho} e^{-\rho \tau_{2}}, \\
\psi(t)=\frac{1}{\rho}+\frac{\rho-r}{r \rho} e^{\rho\left(t-\tau_{2}\right)}, \quad t<\tau_{2} .
\end{gathered}
$$

Подставляем найденное решение в $y_{2}$

$$
y_{2}(\psi(t), t)=r \psi(t)-1=\frac{r}{\rho}\left(1+\frac{\rho-r}{r} e^{\rho\left(t-\tau_{2}\right)}\right)-1=\left(1-\frac{r}{\rho}\right)\left(e^{\rho\left(t-\tau_{2}\right)}-1\right) .
$$

Так как $\rho<r \Rightarrow 1-\frac{r}{\rho}<0$.

На рассматриваемом промежутке

$$
t-\tau_{2}<0 \Rightarrow e^{\rho\left(t-\tau_{2}\right)}<1 \Rightarrow e^{\rho\left(t-\tau_{2}\right)}-1<0 .
$$

В итоге получаем, что $y_{2}(t)>0, t<\tau_{2}$. Пришли к противоречию, значит, данный случай не выполняется.

2. Пусть $y_{2}(\psi(t) t)=0, \quad t<\tau_{2}$. Проверим возможность скользящего режима

$$
y_{2}(\psi, t)=r \psi-1, \text { то } \psi=\frac{1}{r} .
$$

Проведем проверку с помощью дифференцирования по $t$

$$
\frac{d}{d t}(r \psi-1)=0 \Rightarrow r \dot{\psi}=0 \Rightarrow r \neq 0 \Rightarrow \dot{\psi}=0 .
$$

Вернемся к сопряженному уравнению

\section{Baikal Research Journal}




$$
\dot{\psi}=\rho \psi-r \psi v-1+v=0,
$$

так как $v(r \psi-1)=0$, то получаем

$$
\rho \psi-1=0 \Rightarrow \psi=\frac{1}{\rho} \neq \frac{1}{r} .
$$

Пришли к противоречию, значит, данный случай не выполняется.

3. Пусть теперь $y_{2}(\psi(t), t)>0, t<\tau_{2}$. Следовательно, $v^{*}=1$. Подставляем данное управление в сопряженное уравнение

$$
\dot{\psi}=\rho \psi-r \psi(c u+v)-(1-u-v) \quad \psi(T)=0,
$$

получаем

$$
\dot{\psi}=\rho \psi-r \psi=\psi(\rho-r), \quad \psi\left(\tau_{2}\right)=\frac{1}{r} .
$$

Решим поставленную задачу Коши. Уравнение представляет собой однородное линейное дифференциальное уравнение 1-го порядка. Проинтегрировав, находим решение

$$
\psi(t)=\frac{1}{r} e^{(\rho-r)\left(t-\tau_{2}\right)}
$$

Подставляем полученное выражение в $y_{2}(\psi(t), t)$

$$
y_{2}(t)=e^{(\rho-r)\left(t-\tau_{2}\right)}-1 \text {. }
$$

Так как $0<\rho<r$ и $t<\tau_{2}$, то $(\rho-r)\left(t-\tau_{2}\right)>0$. Тогда

$$
e^{(\rho-r)\left(t-\tau_{2}\right)}-1>0
$$

отсюда получаем $y_{2}(t)>0, \quad 0 \leq t<\tau_{2}$.

Это не противоречит нашим предположениям, следовательно, данный случай выполняется. Ваким образом, в момент времени $\tau_{2}=T-\frac{1}{\rho} \ln \left(\frac{r}{r-\rho}\right)$ сопряженная траектория
имеет вид

$$
\psi(t)=\frac{1}{r} e^{(\rho-r)\left(t-\tau_{2}\right)} .
$$

С учетом этого

$$
y_{1}(\psi(t), t)=c e^{(\rho-r)\left(t-\tau_{2}\right)}-1 .
$$

Найдем новую точку переключения $y_{1}(\psi(t), t)=0$

$$
\begin{gathered}
c e^{(\rho-r)\left(t-\tau_{2}\right)}=1, \\
e^{(\rho-r)\left(t-\tau_{2}\right)}=\frac{1}{c} .
\end{gathered}
$$

Прологарифмировав это выражение, получим

$$
(\rho-r)\left(t-\tau_{\curvearrowright}\right)=-\ln c .
$$

отсюда $t=\tau_{2}-\frac{1}{\rho-r} \ln c$. Обозначим через $\tau_{3}=\tau_{2}-\frac{1}{\rho-r} \ln c$ точку переключения. В точке $t=\tau_{3} \quad y_{1}(t)=0$, при $\tau_{3}<t \leq T \quad y_{1}(t)<0$.

\section{Baikal Research Journal}


Найдем знак $y_{1}(t)$ в зависимости от расположения $\tau_{3}$.

1) Если $\tau_{3} \leq 0$, то $y_{1}(t)<0, \quad 0 \leq t \leq T$. Значит

$$
\begin{gathered}
u^{*}=0, \quad t \in[0 ; T], \\
v^{*}= \begin{cases}1, & 0 \leq t<\tau_{2} \\
0, & \tau_{2} \leq t \leq T\end{cases}
\end{gathered}
$$

2) Если $0<\tau_{3}<T$, то сделаем предположения на знак $y_{1}(\psi)$ на участке $\left(0 ; \tau_{3}\right]$

1. $y_{1}(\psi)<0$ на $0<t<\tau_{3}$.

2. $y_{1}(\psi)=0$ на $0<t<\tau_{3}$.

3. $y_{1}(\psi)>0$ на $0<t<\tau_{3}$.

Далее проведем анализ этих ситуаций.

Пусть $y_{1}(\psi(t) t)<0, t<\tau_{3}$. Следовательно, $u^{*}=0$. Подставляем данное управление в сопряженное уравнение и находим его решение

$$
\dot{\psi}=\rho \psi-r \psi=\psi(\rho-r), \quad \psi\left(\tau_{3}\right)=\frac{1}{r c} .
$$

Проинтегрировав, получим

$$
\begin{gathered}
\psi(t)=c_{2} e^{(\rho-r) t}, \\
\psi\left(\tau_{3}\right)=\frac{1}{r c} \Rightarrow c_{2}=\frac{1}{r c} e^{-(\rho-r) \tau_{3}}, \\
\psi(t)=\frac{1}{r c} e^{(\rho-r)\left(t-\tau_{3}\right)} .
\end{gathered}
$$

Подставляем найденное решение в выражение для $y_{1}(\psi, t)$

$$
y_{1}(t)=e^{(\rho-r)\left(t-\tau_{3}\right)}-1
$$

Так как $\rho-r<0$, и на рассматриваемом промежутке $t-\tau_{3}<0$, то

$$
e^{(\rho-r)\left(t-\tau_{3}\right)}>1
$$

В итоге получаем, что $y_{1}(t)>0, t<\tau_{3}$. Пришли $к$ противоречию, значит, данный случай не выполняется

2. Пусть $y_{1}(\psi(t), t)=0, \quad t<\tau_{3}$. Проверим возможность скользящего режима

$$
y_{1}(\psi)=r c \psi-1, \text { тогда } \psi=\frac{1}{r c} \text {. }
$$

Проведем проверку с помощью дифференцирования по $t$

Вернемся к сопряженному уравнению

$$
\frac{d}{d t}(r c \psi-1)=0 \Rightarrow r c \dot{\psi}=0 \Rightarrow \begin{aligned}
& r \neq 0 \\
& c \neq 0
\end{aligned} \Rightarrow \dot{\psi}=0 .
$$

$$
\begin{gathered}
\dot{\psi}=\rho \psi-r \psi(c u+1)-(1-u-1), \\
(\rho-r) \psi+u(1-r c \psi)=0,
\end{gathered}
$$

Так как $y_{1}(\psi)=r c \psi-1$, то получаем

\section{Baikal Research Journal}




$$
(\rho-r) \psi=0 \Rightarrow \psi=0 \neq \frac{1}{r c} .
$$

Пришли к противоречию, значит, данный случай не выполняется.

3. Пусть теперь $y_{1}(\psi(t), t)>0, t<\tau_{3}$. Следовательно, $u^{*}=\frac{g-r}{c r}$.

Подставляем данное управление в сопряженное уравнение

$$
\dot{\psi}=\rho \psi-r \psi(c u+v)-(1-u-v),
$$

получаем

$$
\dot{\psi}=\psi(\rho-g)+\frac{g-r}{c r}, \quad \psi\left(\tau_{3}\right)=\frac{1}{c r} .
$$

Решим поставленную задачу Коши. Уравнение представляет собой неоднородное линейное дифференциальное уравнение 1-го порядка, его решение находится по стандартной формуле.

$$
\psi(t)=\frac{\rho-r}{c r(\rho-g)} e^{(\rho-g)\left(t-\tau_{3}\right)}-\frac{g-r}{c r(\rho-g)} .
$$

Подставляем полученное выражение в $y_{1}(\psi(t), t)$

$$
y_{1}(t)=\frac{\rho-r}{\rho-g} e^{(\rho-g)\left(t-\tau_{3}\right)}-\frac{\rho-r}{\rho-g}=\frac{\rho-r}{\rho-g}\left(e^{(\rho-g)\left(t-\tau_{3}\right)}-1\right) .
$$

Рассмотрим знак $y_{1}(t)$ в зависимости от знака выражения $(\rho-g)$

а) если $(\rho-g)<0$, то $\left(e^{(\rho-g)\left(t-\tau_{3}\right)}-1\right)>0$, тогда учитывая, что $(\rho-r)<0$ по условию задачи, получаем $y_{1}(t)>0$.

b) если $(\rho-g)>0$, то $\left(e^{(\rho-g)\left(t-\tau_{3}\right)}-1\right)<0$, тогда учитывая, что $(\rho-r)<0$ по условию задачи, получаем $y_{1}(t)>0$.

В итоге, получаем $y_{1}(t)>0, t<\tau_{3}$.

Это не противоречит нашим предположениям, следовательно, данный случай выполняется. Таким образом, получили, что в момент времени $\tau_{3}=\tau_{2}-\frac{1}{\rho-r} \ln c$ сопряженная
траектория имеет вид

$$
\psi(t)=\frac{\rho-r}{\operatorname{cr}(\rho-g)} e^{(\rho-g)\left(t-\tau_{3}\right)}-\frac{g-r}{c r(\rho-g)} .
$$

С учетом этого

$$
y_{2}(t)=\frac{\rho-r}{c(\rho-g)} e^{(\rho-g)\left(t-\tau_{3}\right)}-\frac{g-r}{c(\rho-g)}-1
$$

Найдем следующую точку переключения $y_{2}(\psi(t), t)=0$ и обозначим ее через

$$
\tau_{4}=\tau_{3}+\frac{1}{\rho-g} \ln \begin{gathered}
g-r+c \rho-c g \\
\rho-r
\end{gathered} .
$$

Так как $\tau_{4}>\tau_{3}$, то точек переключения больше нет.

В результате получили экстремальные управления

1. Если $\tau_{2} \leq 0$, то есть

$$
T \leq{ }_{\rho}^{1} \ln \frac{r}{r-\rho},
$$

\section{Baikal Research Journal}




$$
\left\{\begin{array}{l}
u^{*}(t)=0 \\
v^{*}(t)=0
\end{array}, \quad t \in[0 ; T] .\right.
$$

Соответствующая фазовая траектория $x^{*}(t)=x_{0}$. тогда

2. Если $\tau_{2}>0$, то есть $T>\frac{1}{\rho} \ln \frac{r}{r-\rho}$, но $\tau_{3} \leq 0$, то есть $T \leq \frac{1}{\rho-r} \ln c+\frac{1}{\rho} \ln \frac{r}{r-\rho}$,

$$
\begin{aligned}
& u^{*}(t)=0, \\
& v^{*}(t)=\left\{\begin{array}{ll}
1, & 0 \leq t<\tau_{2} \\
0, & \tau_{2} \leq t \leq T
\end{array} .\right.
\end{aligned}
$$

Соответствующая фазовая траектория

$$
x^{*}(t)=\left\{\begin{array}{cc}
x_{0} e^{r t}, & 0 \leq t<\tau_{2} \\
x_{0} e^{g \tau_{2}}, & \tau_{2} \leq t \leq T
\end{array} .\right.
$$

3. Если $\tau_{3}>0$, то есть $T>\frac{1}{\rho-r} \ln c+\frac{1}{\rho} \ln \frac{r}{r-\rho}$, тогда

$$
\begin{gathered}
u^{*}(t)=\left\{\begin{array}{cc}
g-r & 0 \leq t<\tau_{3}, \\
c r & \tau_{3} \leq t \leq T \\
0, &
\end{array}\right. \\
v^{*}(t)=\left\{\begin{array}{cc}
1, & 0 \leq t<\tau_{2} \\
0, & \tau_{2} \leq t \leq T
\end{array}\right.
\end{gathered}
$$

Соответствующая фазовая траектория

$$
x^{*}(t)=\left\{\begin{array}{cc}
x_{0} e^{g t}, & 0 \leq t<\tau_{3} \\
x_{0} e^{(g-r) \tau_{3}+r t}, & \tau_{3} \leq t<\tau_{2} . \\
x_{0} e^{(g-r) \tau_{3}+r \tau_{2}}, & \tau_{2} \leq t \leq T
\end{array}\right.
$$

Проводя аналогичные рассуждения в случае Б получим экстремальные управления вида:

1. Если $\tau_{1} \leq 0$, то есть $T \leq \frac{1}{\rho} \ln \left(\frac{r}{r-\rho}\right)$, тогда

$$
\left\{\begin{array}{l}
u^{*}(t)=0 \\
v^{*}(t)=0
\end{array}, \quad t \in[0 ; T] .\right.
$$

Соответствующая фазовая траектория

$$
x^{*}(t)=x_{0} .
$$

Если $\tau_{1}>0$, то есть $T>\frac{1}{\rho} \ln \left(\frac{r}{r-\rho}\right)$, тогда

\section{Baikal Research Journal}




$$
\begin{aligned}
& u^{*}(t)=0, \\
& v^{*}(t)= \begin{cases}\frac{g}{r}, & 0 \leq t<\tau_{1} . \\
0, & \tau_{1} \leq t \leq T\end{cases}
\end{aligned}
$$

Соответствующая фазовая траектория

$$
x^{*}(t)=\left\{\begin{array}{cl}
x_{0} e^{g t}, & 0 \leq t<\tau_{1} \\
x_{0} e^{g \tau_{1}}, & \tau_{1} \leq t \leq T
\end{array}\right.
$$
чая A:

Приведем экономическую интерпретацию оптимального управления для слу-

$$
\text { 1. } u^{*}(t)=0, v^{*}(t)=0 \text { на всем отрезке, если } T \leq \frac{1}{\rho} \ln \frac{r}{r-\rho} \text {. }
$$

В этом случае наиболее выгодно ничего не предпринимать: ни привлекать, ни размещать капитал.

$$
u^{*}(t)=0, v^{*}(t)=\left\{\begin{array}{ll}
1, & 0 \leq t<\tau_{2} \\
0, & \tau_{2} \leq t \leq T
\end{array}, \text { если } \tau_{3}<0, \tau_{2}>0\right.
$$

Если $T \in\left(\frac{1}{\rho} \ln \left(\frac{r}{r-\rho}\right) ; \frac{1}{\rho} \ln \left(\frac{r}{r-\rho}\right)+\frac{1}{\rho-r} \ln c\right)$, то в этом случае привлекать дополнительные средства нецелесообразно на всем периоде планирования. Все собственные средства подлежат размещению в первом периоде планирования, а во втором периоде при $t \in\left[T-\frac{1}{\rho} \ln \left(\frac{r}{r-\rho}\right) ; T\right]$ размещать собственные средства не выгодно.

$$
\text { 2. } u^{*}(t)=\left\{\begin{array}{cc}
\frac{g-r}{r c}, & 0 \leq t<\tau_{3} \\
0, & \tau_{3} \leq t \leq T
\end{array}, \quad v^{*}(t)=\left\{\begin{array}{cc}
1, & 0 \leq t<\tau_{2} \\
0, & \tau_{2} \leq t \leq T
\end{array} \text {, если } \tau_{3}>0\right.\right.
$$

Если $T>\frac{1}{\rho} \ln \left(\frac{r}{r-\rho}\right)+\frac{1}{\rho-r} \ln c$, то на первом этапе выгодно привлекать капитал извне так, чтобы он составлял $\frac{g-r}{r c}$ часть собственного. Кроме того, выгодно разместить все свободные средства (например, выдав кредит). Во втором периоде нужно продолжать вкладывать собственный капитал для получения прибыли, но привлекать ресурсы извне уже не рекомендуется. В последнем периоде остается только ждать прибыли, которая максимизируется благодаря правильно выбранным стратегиям на ранних этапах работы.

Приведем экономическую интерпретацию оптимального управления для случая Б:

$$
u^{*}(t)=0, v^{*}(t)=0 \text { на всем отрезке, если } T \leq \frac{1}{\rho} \ln \left(\frac{r}{r-\rho}\right) .
$$

В этом случае выгоднее ничего не предпринимать: ни привлекать, ни размещать капитал.

$$
u^{*}(t)=0, v^{*}(t)=\left\{\begin{array}{cc}
\frac{g}{r}, & 0 \leq t<\tau_{1} \\
0, & \tau_{1} \leq t \leq T
\end{array}, \text { если } T>\frac{1}{\rho} \ln \left(\frac{r}{r-\rho}\right)\right.
$$

\section{Baikal Research Journal}


В этом случае привлекать дополнительные средства нецелесообразно на всем периоде планирования. Доля собственных средств, подлежащих размещению, в первом периоде должна составлять $\frac{g}{r}$, а во втором периоде при $t \in\left[T-\frac{1}{\rho} \ln \left(\frac{r}{r-\rho}\right) ; T\right]$ размещать собственные средства не выгодно.

\section{Список использованной литературы}

1. Sakswa Y. On global convergence of an algorithm for optimal control / Y. Saksawa, Y. Shindo // IEEE Transactions on Automatic Control. — 1980. - Vol. AC-25, № 6. P. 1149-1158.

2. Sethi S. P. Optimal control theory. Application to management science / S. P. Sethi, G. L. Thomson. - Boston : Martinus Nijhoff Publ. Co., 1981. - 481 p.

3. Антипина Н. В. Линейные функции Ляпунова-Кротова и достаточные условия оптимальности в форме принципа максимума / Н. В. Антипина, В. А. Дыхта // Известия высших учебных заведений. Математика. - 2002. - Т. 46, № 12. - С. 9-20.

4. Аргучинцев А. В. Оптимальное управление: нелокальные условия, вычислительные методы и вариационный принцип максимума / А. В. Аргучинцев, В. А. Дыхта, В. А. Срочко // Известия высших учебных заведений. Математика. - 2009. - Т. 53, № 1. - C. 3-43.

5. Кротов В. Ф. Методы и задачи оптимального управления / В. Ф. Кротов, В. И. Гурман. - М. : Наука, 1973. - 446 с.

6. Срочко В. А. Итерационные методы решения задач оптимального управления / В. А. Срочко. - М. : Физматлит, 2000. - 160 с.

7. Срочко В. А. Достаточные условия оптимальности экстремальных управлений на основе формул приращения функционала / В. А. Срочко, В. Г. Антоник // Известия высших учебных заведений. Сер.: Математика. - 2014. - Т. 58, № 8. - С. 96-102.

8. Срочко В. А. Достаточные условия оптимальности в задачах управления на основе формул приращения функционалов / В. А. Срочко, В. Г. Антоник, Е. В. Аксенюшкина // Известия Иркутского государственного университета. Сер.: Математика. — 2014. T. 8. - C. $125-140$.

9. Аксенюшкина Е. В. Математика-2. Нелинейное и линейное программирование / Е. В. Аксенюшкина, Н. В. Тарасенко, С. В. Тимофеев. - Иркутск : Изд-во БГУЭП, 2009. $124 \mathrm{c}$.

10. Аксенюшкина Е. В. К численному решению задач оптимального управления с функциональными ограничениями / Е. В. Аксенюшкина // Оптимизация, управление, интеллект. - 1999. - № 3. - С. 152-156.

11. Необходимые условия в оптимальном управлении / А. П. Афанасьев [и др.]. - М. : Наука, 1990. - 319 с.

12. Аксенюшкина Е. В. Построение оптимальной инвестиционной политики фирмы / Е. В. Аксенюшкина // Известия Байкальского государственного университета. - 2017. T. 27, № 2. - C. 274-280.

\section{References}

1. Sakswa Y., Shindo Y. On global convergence of an algorithm for optimal control. IEEE Transactions on Automatic Control, 1980, vol. AC-25, no. 6, pp. 1149-1158.

2. Sethi S. P., Thomson G. L. Optimal control theory. Application to management science. Boston : Martinus Nijhoff Publ. Co., 1981. 481 p.

3. Antipina N. V., Dykhta V. A. Linear Lyapunov-Krotov functions and sufficient conditions for optimality in the form of maximum principle. Izvestiya Vysshikh uchebnykh zavedenii. Matematika = Bulletin of Higher Educational Institutions. Mathematics, 2002, vol. 46, no. 12, pp. 9-20. (In Russian).

4. Arguchintsev A. V., Dykhta V. A., Srochko V. A. Optimal control: nonlocal conditions, computational methods, and the variational principle of maximum. Izvestiya Vysshikh uchebnykh zavedenii. Matematika = Bulletin of Higher Educational Institutions. Mathematics, 2009, vol. 53, no. 1, pp. 3-43. (In Russian).

\section{Baikal Research Journal}


5. Krotov V. F., Gurman V. I. Metody i zadachi optimal'nogo upravleniya. [Optimal Control Methods and Problems]. Moscow, Nauka Publ., 1973. 446 p.

6. Srochko V. A. Iteratsionnye metody resheniya zadach optimal'nogo upravleniya. [Iterative methods for solving optimal control problems]. Moscow, Fizmatlit Publ., 2000. 160 p.

7. Srochko V. A., Antonik V. G. Sufficient conditions for optimality in control problems based on functional excess formulas. Izvestiya Vysshikh uchebnykh zavedenii. Matematika= Bulletin of Higher Educational Institutions. Mathematics, 2014, vol. 58, no. 8, pp. 96-102. (In Russian).

8. Srochko V. A., Antonik V. G., Aksenyushkina Ye. V. Sufficient conditions for optimality in control problems based on functional excess formulas. Vestnik Irkutskogo gosudarstvennogo universiteta. Seriya Matematika = Bulletin of Irkutsk State University. Series Mathematics, 2014, vol. 8, pp. 125-140. (In Russian).

9. Aksenyushkina Ye. V., Tarasenko N. V., Timofeyev S. V. Matematika-2. Nelineinoe $i$ lineinoe programmirovanie [Mathematics-2. Non-linear and linear programming]. Irkutsk, Baikal State University of Economics and Law Publ., 2009. 124 p.

10. Aksenyushkina Ye. V. On numerical solution of optimal control problems with functional limitations. Optimizatsiya, upravlenie, intellekt = Optimisation, Control, Intelligence, 1999, no. 3, pp. 152-156. (In Russian).

11. Afanasyev A P., Dikusar V. V., Milyutin S. A., Chukanov A. A. Neobkhodimye usloviya $v$ optimal'nom upravlenii [Necessary conditions in optimal control]. Moscow, Nauka Publ., 1990. $319 \mathrm{p}$.

12. Aksenyushkina Ye. V. Formation of an optimal investment policy of company. Izvestiya Baikal'skogo gosudarstvennogo universiteta $=$ Bulletin of Baikal State University, 2017, vol. 27, no 2, pp. 274-280. (In Russian).

\section{Информация об авторе}

Аксенюшкина Елена Владилировна - кандидат физико-математических наук, доцент, кафедра математики и эконометрики, Байкальский государственный университет, 664003, г.Иркутск, ул. Ленина, 11, e-mail: aks.ev@mail.ru.

\section{Author}

Yelena V. Aksenyushkina - PhD in Physics and Mathematics, Associate Professor, Chair of Mathematics and Econometrics, Baikal State University, 664003, Irkutsk, 11 Lenin St.; e-mail: aks.ev@mail.ru.

\section{Для цитирования}

Аксенюшкина Е. В. Нахождение оптимальной инвестиционной стратегии финансовой организации / Е. В. Аксенюшкина // Baikal Research Journal. - 2017. — T. 8, № 4. DOI : $10.17150 / 2411-6262.2017 .8(4) .16$.

\section{For Citation}

Aksenushkina Ye. V. Finding an optimal investment strategy of financial institution. Baikal Research Journal, 2017, vol. 8, no. 4. DOI: 10.17150/2411-6262.2017.8(4).16. (In Russian).

\section{Baikal Research Journal}

